\title{
Hit two birds with one stone: why crystalline glucosamine sulphate used for osteoarthritis medication is beneficial for patients with risk of cardiovascular disorders
}

\author{
Eugeniusz J. Kucharz \\ Department of Internal Medicine, Rheumatology and Clinical Immunology, Medical University of Silesia, Katowice, Poland
}

The latest update of recommendations of management of knee osteoarthritis highlights the role of crystalline glucosamine sulphate in the treatment of osteoarthritis [1]. It results from differentiation of the crystalline form of glucosamine sulphate from other forms of the substance, and superior efficacy of the crystalline form [2]. It has been evidenced that only crystalline glucosamine sulphate is well absorbed and reaches a concentration required for anti-inflammatory activity [3]. This mechanism is believed to be responsible for efficacious therapeutic action of the drug [2]. On the other hand, other medications (paracetamol, non-steroidal anti-inflammatory drugs) are more toxic and their application is associated with an increase in cardiovascular events [4].

Almost concomitantly with publication of the updated recommendations, the paper of Ma et al. appeared [5]. The authors revealed that administration of glucosamine decreases the risk of cardiovascular events. They did not consider the dose, chemical form or period of application of glucosamine.

The aim of this letter is to propose a hypothetical explanation of this phenomenon. Glucosamine is shown to inhibit inflammation due to inhibition of NFkB activation [6]. In this way, glucosamine reduces the level of inflammation in osteoarthritic joints but probably also in other parts of the body, including the arterial wall. Atherosclerosis, the main cause of cardiovascular events, is an inflammatory process. It has been suggested that systemic inflammation enhances atherosclerosis and its sequelae [7]. Reduction of systemic inflammation is hypothesized as an additional beneficial effect of glucosamine but can be achieved in clinical practice only in patients receiving the crystalline form of the drug. It is possible that analysis of the subgroup of patients in the study by Ma et al. [5] treated with crystalline glucosamine sulphate for a sig- nificant period will evidence more advanced reduction of risk of cardiovascular events than that in all patients receiving glucosamine.

The described mechanism of cardiovascular event reduction may also be supplemented by other actions of the drugs (e.g. increase in physical activity due to reduction of articular pain). Most important is however the discovery of cardiovascular event risk reduction as an additional beneficial effect of glucosamine, and further studies in this field are needed.

The author declares no conflict of interest.

\section{References}

1. Bruyère $\mathrm{O}$, Honvo $\mathrm{G}$, Veronese $\mathrm{N}$, et al. An updated algorithm recommendation for the management of knee osteoarthritis from the European Society for Clinical and Economic Aspects of Osteoporosis, Osteoarthritis and Musculoskeletal Diseases (ESCEO). Semin Arthritis Rheum 2019; pii: S0049-0172(19)30043-5 [Epub ahead of print].

2. Kucharz EJ, Kovalenko V, Szántó S, et al. A review of glucosamine for knee osteoarthritis: why patented crystalline glucosamine sulfate should be differentiated from other glucosamines to maximize clinical outcomes. Curr Med Res Opin 2016; 32: 997-1004.

3. Persiani S, Roda E, Rovati LC, et al. Glucosamine oral bioavailability and plasma pharmacokinetics after increasing doses of crystalline glucosamine sulfate in man. Osteoarthritis Cartilage 2005; 13: 1041-1049.

4. Kucharz EJ, Szántó S, Ivanova Goycheva M, et al. Endorsement by Central European experts of the revised ESCEO algorithm for the management of knee osteoarthritis. Rheumatol Int 2019; 39: 1117-1123.

5. Ma H, Li X, Sun D, et al. Association of habitual glucosamine use with risk of cardiovascular disease: prospective study in UK Biobank. BMJ 2019; 365: 11628.

6. Chiusaroli R, Piepoli T, Zanelli T, et al. Experimental pharmacology of glucosamine sulfate. Int J Rheumatol 2011; 2011: 939265.

7. Kucharz EJ. Chronic inflammation-enhanced atherosclerosis: can we consider it as a new clinical syndrome? Med Hypotheses 2012; 78: 396-397. 